\title{
Balkanologie
}

Balkanologie Revue d'études pluridisciplinaires

Vol. IX, n' 1-2 | 2005

Volume IX Numéro 1-2

\section{Gallagher (Tom), The balkans in the new Millennium. In the Shadows of War and Peace}

London / New York : Routledge, 2005, 232 p.

\section{Patrick Michels}

\section{(2) OpenEdition}

\section{Journals}

Édition électronique

URL : http://journals.openedition.org/balkanologie/2001

DOI : 10.4000/balkanologie.2001

ISSN : 1965-0582

Éditeur

Association française d'études sur les Balkans (Afebalk)

Édition imprimée

Date de publication : 1 décembre 2005

ISSN : 1279-7952

\section{Référence électronique}

Patrick Michels, «Gallagher (Tom), The balkans in the new Millennium. In the Shadows of War and Peace », Balkanologie [En ligne], Vol. IX, nº 1-2 | 2005, mis en ligne le 14 janvier 2010, consulté le 17 décembre 2020. URL : http://journals.openedition.org/balkanologie/2001 ; DOI : https://doi.org/ 10.4000/balkanologie.2001

Ce document a été généré automatiquement le 17 décembre 2020.

(c) Tous droits réservés 


\section{Gallagher (Tom), The balkans in the new Millennium. In the Shadows of War and Peace}

London / New York : Routledge, 2005, 232 p.

\section{Patrick Michels}

\section{RÉFÉRENCE}

Gallagher (Tom), The balkans in the new Millennium. In the Shadows of War and Peace, London / New York : Routledge, 2005, 232 p.

Dans ce dernier volume de sa trilogie, T. Gallagher poursuit son investigation sur l'action «occidentale» dans les Balkans. Ce tome est consacré aux crises qui sont survenues dans les Balkans depuis 1998; l'auteur y insiste sur la lenteur de l'implication des pays de l'OTAN, l'adoption de mesures minimalistes pour contenir les problèmes, même si, globalement, l'Occident est mieux préparé face aux crises kosovare (1998) et macédonienne (2001), bien que n'étant pas habitué à traiter avec des États faibles (tel que peut l'être la Bosnie-Herzégovine). En 1999, l'UE est dotée d'une nouvelle équipe qui estime qu'intégrer les pays balkaniques à l'UE représente un moteur pour apaiser les disputes frontalières et normaliser les relations interethniques. Le retrait des États-Unis aboutit à une plus forte implication de l'UE dans le maintien de la paix en Bosnie-Herzégovine, la reconstruction, la restauration des infrastructures et la transformation des institutions. Néanmoins, l'option économique privilégiée (privatisation rapide) favorise ceux qui ont profité de la guerre et ceux qui ont détourné les fonds publics.

2 En Grèce, Papandréou affronte un problème de crédibilité. Chypre, les relations avec la Turquie, lui ont servi à détourner l'attention de la population, tout comme les controverses avec la république de Macédoine. La dispute porte sur le nom du jeune État, revendiqué par la Grèce. Son alliance de fait avec le régime de Milošević est une 
des raisons de l'implication de l'UE dans cette dispute. L'autre sujet de discorde concerne l'Épire du nord. Le Traité de paix de Dayton signé, l'UE exerce plus de pressions sur la Grèce. Cet épisode met en évidence le clivage grec entre le nord du pays (sur lequel l'embargo a des conséquences négatives) et le sud (éloigné de ces préoccupations). Par ailleurs, si les tremblements de terre turc et grec de 1999 rapprochent les deux États, l'affaiblissement de la politique turque dans les Balkans en cette fin de $\mathrm{XX}^{\mathrm{e}}$ siècle n'y est pas étrangère.

Dans le deuxième chapitre, consacré au Kosovo, T. Gallagher revient sur les racines des deux nationalismes qui s'y opposent (centre de l'empire serbe selon l'idéologie nationale établie au XIXe siècle; primauté des Albanais descendants des Illyriens, Prizren, racine du mouvement national albanais). La politique de l'État yougoslave, puis serbe, vis-à-vis des Albanais, a engendré une résistance passive, sous l'égide de Rugova (qui veut bien négocier le statut de la Province sur la base de l'indépendance). Le jeu Rugova / Milošević (« Milošević tolerated Rugova for as long as he kept the Albanian quiet, which provided Rugova with the space for what he believed would be a steady if slow advancement of the cause of the Albanian independence », p. 31) ne satisfait pas tout le monde, et le leader politique ne peut empêcher la création de nouveaux partis. L'«Occident» ne prête que peu d'attention au Kosovo, malgré les multiples avertissements de commissions d'enquête sur les droits de l'Homme, reconnaissant de fait la mainmise de Belgrade sur la Province. Le $1^{\mathrm{er}}$ octobre 1997, une manifestation se déroule à Priština sans le soutien de Rugova. C'est le vecteur de la dissension au sein de la LDK (Ligue Démocratique du Kosovo) et l'émergence ouverte du mouvement armé. Néanmoins, les attaques de l'UÇK (Ushtria Çlirimtare e Kosovës, Armée de libération du Kosovo) depuis 1996 incitent l'« Occident » à réagir. Les déclarations vont dans le sens d'un Kosovo autonome dans la Yougoslavie et, surtout, les négociations se font avec les autorités serbes. La problématique est la même qu'en 1991 sur l'ensemble de la Yougoslavie, chacun refuse de céder devant l'autre. Les négociateurs sont précautionneux, ne voulant pas donner de signes interprétables par les Albanais de Macédoine. Par ailleurs, quelle incidence aurait un Kosovo indépendant sur la BosnieHerzégovine ? Les choix proposés et proposables sont hésitants, entre une partition du Kosovo et une troisième République au sein de la Fédération yougoslave. C'est le massacre de Račak (15 janvier 1999) qui fournit le prétexte à l'« Occident » de s'engager plus activement. Les négociations de Rambouillet, avec cette fois des représentants de l'UÇK et de la LDK, aboutissent, devant le refus serbe de signer les accords, au choix de l'option militaire.

4 Il s'agit du premier conflit armé en Europe depuis 60 ans. Les autorités serbes profitent du bombardement pour opérer des déplacements de la population albanaise du Kosovo par des forces paramilitaires liées au haut commandement militaire. Milošević et l'OTAN ont chacun mal interprété les intentions de l'autre. Le traitement des réfugiés en Albanie révèle que les conditions économiques priment sur la solidarité nationale. En Yougoslavie, le sentiment anti-occidental se développe, d'autant plus que la presse muselée ne rend pas compte des raisons de cette attaque. Le 3 juin 1999, Milošević accepte le retrait serbe du Kosovo qui est divisé en trois zones (nord-américaine, française, allemande, avec des troupes russes dans chacune). Afin de maintenir l'union Serbie-Monténégro, la solution choisie pour le Kosovo a été rapide. La chute de Milošević en 2000 ne met pas fin aux problèmes du pays. L'assassinat de Djindjić révèle 
la polarisation qui existe entre réformateurs et nationalistes et que la mafia, les profiteurs de guerre restent très présents.

5 En Macédoine, des revendications albanaises se font entendre, mais de nombreuses minorités font tampon entre les Macédoniens et les Albanais. Si la cohabitation est difficile, il n'y a pas d'éclatement. Les peurs de grande Albanie se font plus vives avec l'arrivée des réfugiés du Kosovo. Bientôt, la chute des Serbes au Kosovo est un signal pour les Albanais de Macédoine. Le problème devient plus aigu lorsque le ciment externe (Milošević) tombe. En outre, des rumeurs persistantes de corruption, auxquelles s'ajoutent rapidement celles d'un accord entre élites albanaises et macédoniennes sur le partage de l'État, avivent les tensions qui se transforment en combats armés. Ce n'est qu'au bout de trois mois de conflit que l'Occident prend conscience qu'il n'y a de solution sans coût. On peut effectivement se demander si la révolte albanaise n'est pas une révolte d'intégration (mettre fin à l'hégémonie d'un groupe national, cf. p. 109). La communauté internationale parvient à impliquer les minorités, dont les Albanais, dans le processus de décision (d'autant plus que les deux partis nationaux macédoniens luttent entre eux, ce qui les tient à l'écart), la décentralisation semble calmer le jeu mais les partis nationalistes macédonien et albanais attendent peut-être de prendre leur revanche.

Deux États parallèles coexistent en Bosnie-Herzégovine. Néanmoins, en 2002, les hauts représentants de l'ONU (d'abord Wolgang Petrisch [2002-2002], puis Paddy Ashdown [2002-2005]) parviennent à établir de nouvelles Constitutions pour les entités qui se traduisent notamment par un accroissement du pouvoir de l'État central. Ils parviennent également à favoriser le retour des réfugiés (1 million, sur les 2 millions de personnes déplacées, revient sur son lieu d'habitation). Mais la Bosnie-Herzégovine reste un État fracturé, marqué de clientélisme, de corruption et d'économie souterraine et dont le renforcement du centre n'est pas irréversible.

7 Les gestionnaires du Kosovo ont du mal face à la mafia d'Albanie fin 1999. La KFor soutient l'action humanitaire durant l'hiver 1999-2000 et participe à l'effort de reconstruction mais la supériorité de l'UÇK sur la LDK, la distance qu'elle prend avec les institutions du Kosovo, les actes de revanche commis à l'encontre des Serbes et des Tsiganes, la répression contre les Albanais qui dénoncent les membres ou les actes de l'UÇK et l'image des Albanais impliqués dans le crime organisé participent aux hésitations occidentales sur le statut du Kosovo, les États membres de l'UE ne s'entendant pas entre eux. Bien que l'expérience de la Bosnie-Herzégovine ait servi, tout ceci témoigne d'un manque de vision à long terme. L'impréparation de l'UE dans ce conflit a également été patente. Le conflit du Kosovo a néanmoins eu pour conséquence d'inciter à la mise en place d'une politique européenne de sécurité et de défense (1999). Mais l'engagement des forces militaires sur d'autres fronts (en Irak pour le Royaume-Uni, en Afghanistan pour l'Allemagne, en Côte d'Ivoire pour la France) a nuit à sa pleine utilisation. Si la Roumanie et la Bulgarie, en remerciement de leur soutien à l'UE lors de la crise du Kosovo, sont invitées à intégrer l'UE au sommet d'Helsinki en décembre 1999, il n'y a pas de réelle initiative positive prise en vue de cette intégration des Balkans occidentaux. L'UE se trouve en fait démunie face à l'instabilité politique, la corruption et les problèmes économiques exacerbés par l'échec de la Banque mondiale à prioriser la transition économique.

8 La plupart des dirigeants politiques balkaniques s'appuient, depuis 1989, sur des populations tournées, elles, vers des solutions autoritaires. Les modernisateurs - qui se 
trouvent dans les centres urbains - se sont éloignés du champ politique, laissant la voie au crime organisé. L'exemple le plus probant en est la Macédoine en 2001. L'inégalité économique s'est largement accrue et rien n'indique que cette tendance ne va pas s'intensifier. Les alternatives politiques n'ont que peu d'écho, et la fuite des cerveaux n'arrange rien, d'autant plus que les institutions transnationales ont produit des paix «dysfonctionnelles». Même s'il existe des contre-exemples (notamment la Transylvanie), seul l'économique permettra une normalisation des relations ethniques et la protection de la sécurité humaine.

Dans cet ouvrage, T. Gallagher nous montre les incidences des changements de personne dans la direction des États, aux Affaires étrangères, sur la politique choisie vis-à-vis des Balkans. Il insiste sur les conséquences des modifications de contexte et nous rappelle la prédominance de l'économique. Il nous démontre la politique incrémentale occidentale et comment les phrases des diplomates sont sujettes à diverses interprétations. Il n'oublie pas de souligner la corruption de la classe politique balkanique et la prédominance de la mafia. Seule la restauration de l'état de droit, de la loi, permettrait de lutter. L'intégration dans l'UE peut représenter la solution à ces problèmes. 